\title{
PROTOCOLO ANESTÉSICO DENTRO DA DISCIPLINA DE ANESTESIOLOGIA. RELATO DE AULA
}

\author{
Gil Dutra FURTADO ${ }^{1 *}$; Tamara de Moura MENDES ${ }^{2}$; Ellen Monteiro FURTADO; \\ Grazielly Diniz DUARTE; Soraya Abrantes Pinto de BRITO³; Felipe Eduardo da Silva \\ SOBRAL ${ }^{4}$
}

\author{
${ }^{1}$ Graduando em Medicina Veterinária/Centro Universitário Maurício de Nassau (UNINASSAU-PB); Engenheiro \\ Agrônomo/Universidade Federal da Paraíba (UFPB); Doutor em Psicobiologia/Universidade Federal do Rio \\ Grande do Norte (UFRN); Agrônomo-Sócio da Cooperativa de Agronegócio (COOPAGRO), Natal, Rio Grande \\ do Norte, Brasil. \\ ${ }^{2}$ Graduanda em Medicina Veterinária/UNINASSAU-PB; Bióloga/Universidade Federal da Paraíba (UFPB), João \\ Pessoa, Paraíba, Brasil. \\ ${ }^{3}$ Graduando em Medicina Veterinária/UNINASSAU-PB, João Pessoa, Paraíba, Brasil. \\ ${ }^{4}$ Mestre; Médico Veterinário; Professor/UNINASSAU-PB, João Pessoa, Paraíba, Brasil. \\ *Autor para correspondência. E-mail: gdfurtado@ hotmail.com
}

Resumo. Todo profissional veterinário deve ter um bom conhecimento dos fármacos terapêuticos, procurando sempre ter uma atualização constante através de consulta a fontes bibliográficas confiáveis. $\mathrm{Na}$ anestesiologia, sob a temática de protocolo de intervenção para a realização de uma orquiectomia em cães, é abordada as ações que antecedem a intervenção, procurando oferecer ao paciente canino segurança e bem estar. Para atingir este objetivo é necessário a formação adequada dos acadêmicos de medicina veterinária que tem como alvo primordial uma característica generalista, ter desempenho ético, seguro e apropriado para a responsabilidade de lidar com as vidas dos pacientes animais.

Palavras chave: Medicina veterinária; Anestesia; Orquiectomia.

\section{ANESTHETIC PROTOCOL WITHIN THE DISCIPLINE OF ANESTHESIOLOGY. CLASS REPORT}

\begin{abstract}
Every veterinary professional must have a good knowledge of therapeutic drugs, always trying to have a constant update through consultation with reliable bibliographic sources. In anesthesiology, under the theme of intervention protocol for performing an orchiectomy in dogs, the actions that precede the intervention are addressed, seeking to offer the canine patient safety and wellbeing. To achieve this objective, it is necessary to properly train veterinary medicine students, whose primary objective is a generalist characteristic, to have ethical, safe and appropriate performance for the responsibility of dealing with the lives of animal patients.
\end{abstract}

Keywords: Veterinary medicine; Anesthesia; Orchiectomy. 


\section{PROTOCOLO ANESTÉSICO DENTRO DE LA DISCIPLINA DE} ANESTESIOLOGÍA. INFORME DE CLASE

Resumen. Todo profesional veterinario debe tener un buen conocimiento de los medicamentos terapéuticos, procurando siempre tener una actualización constante mediante la consulta con fuentes bibliográficas fiables. En anestesiología, bajo la temática de protocolo de intervención para la realización de una orquiectomía en perros, se abordan las acciones que preceden a la intervención, buscando brindar seguridad y bienestar al paciente canino. Para lograr este objetivo, es necesario capacitar adecuadamente a los estudiantes de medicina veterinaria, cuyo objetivo primordial es una característica generalista, para que tengan un desempeño ético, seguro y adecuado para la responsabilidad de tratar la vida de los pacientes animales.

Palabras clave: Medicina veterinaria; Anestesia; Orquiectomía.

\section{INTRODUÇÃO}

A medicina veterinária é a profissão responsável por manter a saúde dos animais e o bem-estar animal. A palavra Veterinário vem do latim "veterinae", que está relacionado às palavras animal. Assim a medicina veterinária é a ciência médica encarregada de prevenir, diagnosticar e curar as doenças que afetam animais domésticos, animais selvagens e animais de produção (CFMV, 2020).

O conhecimento que o médico veterinário deve ter no encargo da anestesiologia é de grande importância, pois sempre está presente na rotina clínica, cirúrgica, anestésica, dentre outras áreas da medicina veterinária (INCE et al., 2015).

Todo profissional veterinário deve ter um bom conhecimento dos fármacos terapêuticos, procurando sempre ter uma atualização constante através de consulta a fontes bibliográficas confiáveis, além de carregar uma grande responsabilidade profissional (CARDOZO, 2018).

Por isso é requerido do acadêmico em medicina veterinária uma séria atenção na disciplina que trata desta temática. Dentre os fármacos, existem seis principais grupos farmacológicos de importância médico-veterinária, que são compreendidos por antihelmínticos, quimioterápicos e antibióticos, ectoparasiticidas, analgésico/antitérmicos/antiinflamatórios, tranquilizantes/anestésicos e os fármacos com ação hormonal (ANDREI, 2014; LANGELOH, 1996; LUMB; JONES, 2017; YAMAZAKI et al, 2011).

Durante as disciplinas de anestesiologia e farmacologia veterinária, os acadêmicos recebem diversas orientações quanto ao estudo dos mecanismos de ação dos fármacos envolvendo a relação dose e efeito, as vias de administração, a absorção, a distribuição, a 
biotransformação e excreção dos fármacos e a prescrição farmacológica (CARDOZO, 2018; FURTADO; FARIAS; DUARTE, 2019; YAMAZAKI et al., 2011).

São abordados os aspectos envolvidos na aplicação adequada dos fármacos aos animais em relação às indicações, contra indicações e efeitos adversos, em relação a fármacos depressores e estimulantes do sistema nervoso central, fármacos que atuam no sistema nervoso autônomo, fármacos envolvidos com o mecanismo da inflamação e dor, com o sistema cardiovascular, respiratório e eutanásia com seus métodos específicos (CARDOZO, 2018; FURTADO; FARIAS; DUARTE, 2019).

Dentro destes, este artigo irá abordar a aula de anestesiologia veterinária, sob a temática de protocolo na intervenção por meio da orquiectomia em cães, tema este abordado durante a disciplina aplicada no ano de 2020, no Centro Universitário Maurício de Nassau (UNINASSAU - PB) (FURTADO; FARIAS; DUARTE, 2019; SILVA et al., 2019).

\section{RELATO DA AULA}

Com o objetivo de formar profissionais para desempenharem, com ética e qualidade, funções nas áreas de competência da Medicina Veterinária, os educadores abordaram com os educandos os passos para a realização do procedimento de intervenção em cães, para a realização de orquiectomia em cães (CARDOZO, 2018; SILVA et al., 2019).

Para que seja realizada a orquiectomia, antes de tudo é importante que se tenha acesso ao histórico do paciente, as principais afecções, alterações que o paciente apresenta atualmente e as que ele já apresentou outrora, bem como as alterações de exame clínico no qual o mesmo demonstre. Posteriormente deve-se solicitar exames complementares, esses darão maior esclarecimento sobre o caso em questão, para assim traçar um plano anestésico, decidir quais fármacos usar e reduzir ao máximo o risco do procedimento, aumentando assim as chances de sucesso da intervenção (SILVA et al., 2019).

Para garantir o bem-estar do paciente, o médico veterinário deverá propor um protocolo de medicação, prevenindo os riscos inerentes a qualquer procedimento da anestesia veterinária. Neste momento a avaliação pré-anestésica irá ponderar o estado de saúde do paciente canino, favorecendo o diagnóstico de doenças não percebidas pelo médico veterinário, e que teriam o potencial de causar complicações indesejadas durante a anestesia (FURTADO; ARAÚJO, 2019; LUMB; JONES, 2017; YAMAZAKI et al., 2011).

Outro objetivo da avaliação pré-anestésica e a de examinar os riscos que o paciente poderá estar submetido, antevendo as ações necessárias, viabilizando os passos a seguir que sejam mais adequados para o paciente, visto que determinados anestésicos podem agravar 
certas doenças ou desordens metabólicas (FURTADO; ARAÚJO, 2019; LUMB; JONES, 2017).

Para que a avaliação pré-anestésica seja realizada coerentemente, é necessário realizar uma consulta prévia, adquirindo informações relevantes sobre o paciente com o tutor, concedendo ao médico veterinário conhecimentos sobre a idade, raça, temperamento, características especiais do animal, a rotina diária do animal, o estado de saúde física e mental, entre outros. Estas informações são muito importantes, pois, o tutor conhece melhor da vida do seu animal quando comparado ao médico veterinário (FURTADO; ARAÚJO, 2019; LUMB; JONES, 2017; YAMAZAKI et al., 2011).

O médico veterinário deve realizar um exame físico geral, levando em consideração as diferenças e particularidades de cada espécie e/ou raça. Animais pediátricos ou geriátricos têm requisitos anestésicos diferentes dos adultos, e os obesos podem ter mais problemas de oxigenação e respiração (YAMAZAKI et al., 2011).

É recomendado a realização de hemograma completo com pesquisa para parasitos sanguíneos, junto com o perfil bioquímico antes dos procedimentos que envolvam anestesia veterinária, visando detectar anemias, infecções, alterações nas funções dos órgãos, entre outras informações importantes (LUMB; JONES, 2017; YAMAZAKI et al., 2011).

Dependendo dos resultados obtidos com o histórico clínico, exame clínico e análises laboratoriais, ainda poderá ser solicitado a realização de mais exames para completar a avaliação pré-anestésica, como: eletrocardiogramas, radiografias, ecodopplergrafias, mensuração da pressão arterial e/ou análises endócrinas (FURTADO; ARAÚJO, 2019; LUMB; JONES, 2017; YAMAZAKI et al, 2011).

A avaliação pré-anestésica é uma forma de conferir segurança aos pacientes durante procedimentos cirúrgicos e garantir o bem-estar contínuo do animal. Durante a aula, objetivando exemplificar uma ação médica veterinária quanto a anestesiologia, o professor idealizou uma situação onde o profissional irá anestesiar uma paciente, jovem, saudável, de 2 anos, canino, sem raça definida, macho, para um procedimento de orquiectomia eletiva, onde o mesmo pesa $15 \mathrm{~kg}$, não apresentando alterações em hemograma, nem em avaliação clínica e anamnese.

Baseado nestes dados, a classificação de risco do paciente (ASA) seria ASA I, pois o paciente tem todas as condições necessárias para ser submetido a esta cirurgia com pouco risco de vida. O protocolo de medicação pré-anestésica (MPA) a ser utilizada neste paciente seria a analgesia com opioides (morfina) e a sedação com acepromazina e/ou benzodiazepínicos (diazepam), e utilizando como bloqueio local intratesticular a lidocaína. 
Estes fármacos são os indicados para este tipo de intervenção, e o meio de administração no paciente para emprego da MPA, por via intravenosa, seria pela veia cefálica devido a facilidade e eficiência que este vaso proporciona. O fármaco indicado para induzir o paciente seria os dissociativos cetamina e tiletamina (por via intravenosa) e associado com midazolam.

Durante o procedimento cirúrgico, para que o paciente fique seguro e receba uma boa oxigenação, ao entubar, deve-se verificar se acidentalmente não foi realizado manobra em falsa via, levando assim oxigênio ao estômago no lugar dos pulmões, sendo importante constatar se haverá ou não refluxo de gases pulmonares do paciente pela sonda endotraqueal. A sonda deve ser colocada antes da carina da traquéia, pois ao se colocar depois, o ar só irá para um dos pulmões.

É importante também, fazer o teste do balonete para averiguar se o mesmo não está furado ou danificado, este realizado antes da intubação no paciente. Sempre deve-se ficar atento a possíveis obstruções na sonda e lembrar de desinflar o balonete antes de retirar a sonda do paciente, no intuito de não gerar nenhum tipo de traumatismo nas estruturas anatômicas envolvidas. Visando a manutenção anestésica, deve-se utilizar a anestesia inalatória com desflurano.

Com estes procedimentos acima, o médico veterinário poderá realizar a intervenção de forma segura e adequada, tendo o paciente em um estado seguro e com analgesia satisfatória (FURTADO; ARAÚJO, 2019; LUMB; JONES, 2017; YAMAZAKI et al., 2011).

O professor, ao término da aula, ressaltou a importância de os acadêmicos sempre estarem atentos as atualizações técnicas que são obtidas por meio de artigos científicos nas diversas revistas e periódicos, procurando ampliar as técnicas e metodologias que são empregadas em cirurgias e intervenções de orquiectomia bem como das várias outras ações que são inerentes aos médicos veterinários (FURTADO; FARIAS; DUARTE, 2019; LUMB; JONES, 2017).

\section{CONCLUSÕES}

A formação adequada dos acadêmicos de medicina veterinária é o alvo primordial para que estes futuros profissionais tenham um desempenho ético, seguro e apropriado para a responsabilidade de lidar com as vidas dos pacientes animais.

Neste artigo foi possível observar o resultado de uma aula na orientação de ações que envolvem atos que devem ser realizados antes e durante o procedimento de uma intervenção cirúrgica de orquiectomia em um paciente canino de 2 anos e que tem um bom antecedente de saúde. 


\section{REFERÊNCIAS}

ANDREI (Editora). Compêndio Veterinário: Dicionário Brasileiro de Medicamentos Veterinários. 36. ed. São Paulo: Organização Andrei Editora Ltda. 2014.

CARDOZO, H.G. Anestesiologia veterinária. Trabalho de Conclusão de Curso (Graduação em Medicina Veterinária), da Universidade Federal de Santa Catarina - UFSC. Curitibanos. p. 56. 2018.

CFMV. Áreas de atuação do médico-veterinário. Conselho Federal de Medicina Veterinária. Disponível em: http://portal.cfmv.gov.br/portal/pagina/index/id/67/secao/5. Acesso em: 25 abr. 2020.

FURTADO, D.M.; ARAÚJO, R.A. Procedimentos anestésicos utilizados nas emergências de cães e gatos atendidos no Hovet no período de janeiro de 2018 a janeiro de 2019 . Trabalho de Conclusão de Curso (Graduação em Medicina Veterinária - Universidade Federal Rural da Amazônia). BELÉM - PA. 48p. 2019.

FURTADO, G.D.; FARIAS, L.A.; DUARTE, G.D. Mapa mental e a disciplina de imunologia animal na perspectiva da psicopedagogia em medicina veterinária: Revisão Literária. Environmental Smoke, João Pessoa, v. 2, n. 3, p. 52-61, 2019.

INCE, I.; AKSOY, M.; AHISKALIOGLU, A.; COMEZ, M.; DOSTBIL, A.; CELIK, M.; YILMAZ, I.; MAMMADOV, R.; DOGAN, H.; BOZTOK, O.B.; ALTUNER, D. A Comparative Investigation of the Analgesic Effects of Metamizole and Paracetamol in Rats. Journal of Investigative Surgery, London, v. 28, n. 3, p. 173-180, 2015.

LANGELOH, A. A prescrição racional em medicina veterinária e os nomes dos fármacos dos medicamentos alopáticos. Ciência Rural, Santa Maria, v. 26, n. 2, p. 315-321, 1996.

LUMB, W.; JONES, W. Anestesiologia e Analgesia em Veterinária. 5. ed. São Paulo: Roca, 2017. 
SILVA, G.M.F.; FURTADO, G.D.; AMORIM, B.M.; TELLES, J.A.; COSTA, M.A.J.; SOBRAL, F.E.S. Controle de la population de chiens dans la municipalite de João Pessoa (Brésil) pendant les quatre ans de 2015 a 2018. Environmental Smoke, João Pessoa, v. 2, n. 1, p. 79, 2019.

YAMAZAKI, M.S.; MAIA FILHO, A.; DE NARDO, C.D.D.; DE AZEVEDO, R.A. Analgesia e anestesia em procedimentos ortopédicos de pequenos animais. Veterinária Notícias, Uberlândia, v. 17, n. 2, p. 77-89, 2011. 